\title{
Multi-frame speckle reduction in OCT using supercontinuum pumped by noise-like pulses
}

\author{
Chengming Wang***, Yi-Jing You ${ }^{\dagger}$, Shengnan Ai*, Wenxin Zhang*, \\ Wenchao Liao*, Xiao Zhang ${ }^{\S}$, Juicheng Hsieh*, Ning Zhangף,

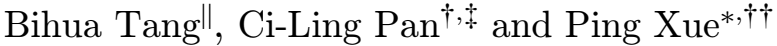 \\ *State Key Laboratory of Low-Dimensional Quantum Physics \\ Department of Physics, Tsinghua University \\ Collaborative Innovation Center of Quantum Matter \\ Beijing, P. R. China \\ $\dagger$ Department of Physics, National Tsing Hua University \\ No. 101, Sec. 2, Kuang-Fu Rd, Hsinchu, Taiwan \\ \$Department of Physics and Institute of Photonics Technologies \\ National Tsing Hua University, Taiwan \\ ${ }^{\S}$ School of Life Science \\ Beijing Institute of Technology, Beijing, P. R. China \\ IInstitute of Forensic Science Ministry of Public Security \\ Beijing, P. R. China \\ "Southwest Medical University \\ Luzhou, Sichuan Province, P. R. China \\ **Nuctech Company Limited, Beijing, P. R. China \\ †xuep@tsinghua.edu.cn
}

Received 26 November 2018

Accepted 17 February 2019

Published 14 March 2019

\begin{abstract}
In most coherent imaging modality, speckle noise is a major cause that blurs the boundary of tissues and degrades the image contrast. By utilizing the unique properties of supercontinuum (SC) generated by noise-like pulses (NLPs) and a simple multi-frame averaging technique, we achieved significant speckle reduction in spectral domain optical coherence tomography (SD-OCT). We quantitatively compared the speckle of our proposed method with those of conventional swept source OCT (SS-OCT) and SD-OCT based on commercial light sources. The experimental results show that SC pumped by NLPs combined with noncoherent averaging method achieves better denoising performance in terms of contrast to noise ratio (CNR).
\end{abstract}

\footnotetext{
†Corresponding author.
}

This is an Open Access article published by World Scientific Publishing Company. It is distributed under the terms of the Creative Commons Attribution 4.0 (CC-BY) License. Further distribution of this work is permitted, provided the original work is properly cited. 
Keywords: Noise-like pulses; supercontinuum generation; speckle reduction; optical coherence tomography.

\section{Introduction}

Optical coherence tomography (OCT) is an emerging biomedical imaging modality based on optical interferometric detection, which achieves noninvasive, high-resolution and cross-sectional imaging. However, like other laser imaging modalities, OCT images contain speckles that depend not only on the wavelength and coherent properties of imaging beam but also on the structure of imaging objects. ${ }^{1}$ Numerous methods have been developed to suppress the speckle noise and improve the image quality. Hardware-based techniques, including spatial, ${ }^{2}$ angular, ${ }^{3,4}$ strain, ${ }^{5}$ and frequency compounding, ${ }^{6,7}$ can effectively reduce the speckle noise while preserving weak structural signal. On the other hand, these approaches usually require complicated optical design and setup. Software-based techniques, including digital filters, ${ }^{8}$ wavelet-based method, ${ }^{9}$ real-time dual window method, ${ }^{10}$ anisotropic diffusion-based method, ${ }^{11}$ and probabilitybased method, ${ }^{12,13}$ have also been developed rapidly and can achieve speckle suppression effectively. Suitable quantization method may also significantly increase contrast, signal-to-noise ratio (SNR) and visual fineness of the OCT image. ${ }^{14}$ Nonetheless, most post-processing algorithms require prior knowledge of the imaging object and may suffer from resolution degradation or artifacts. ${ }^{15}$ In addition, some algorithms are complicated and time consuming, ${ }^{16}$ incapable of real-time high speed OCT imaging.

Light source based on supercontinuum (SC) generation provides extremely broad bandwidth and high output power. Thus, SC is particularly suitable for ultrahigh resolution OCT system. SC is usually generated by using ultrashort pulses through nonlinear phenomena in materials, e.g., optical fibers. Noise-like pulses (NLPs), caused by the soliton collapse effect in the lasers, ${ }^{17}$ have the advantage of broader and smoother spectrum (usually of several tens of $\mathrm{nm}$ ) compared with conventional mode-locked short pulses. ${ }^{18}$ In the time domain, the NLP pulse train consists of relatively long (sub-ns), periodic wave packets, which exhibit a fine inner structure of sub-ps pulses with randomly varying amplitude and duration from one wave packet to the next. Typically, the averaged intensity autocorrelation trace of NLPs is doublescaled with a sub-ps peak riding a wide sub-ns pedestal. Since the NLP-based light source is random in nature, its output exhibits low temporal and spatial coherence. This unique property is very helpful to solve the speckle problem of OCT imaging. It has been reported that amplified NLPs could be successfully used for SC generation with standard single-mode fibers. ${ }^{19}$ In our previous work, ${ }^{20}$ we have also demonstrated the feasibility of SC pumped by NLPs as the light source for ultrahigh resolution spectral domain OCT (SD-OCT) imaging.

In this paper, we present a simple multi-frame averaging method for speckle reduction by utilizing the inherent coherence properties of the SC generated by noise-like pulsed fiber laser. Our results demonstrate that this unique light source has very efficient denoising performance in terms of contrast to noise ratio (CNR) and equivalent number of looks (ENL) and cross-correlation (XCOR) value.

\section{Principles and Experimental Setup}

\subsection{Experimental setup}

As shown in Fig. 1, we constructed an amplified allnormal dispersion (ANDi) Yb-doped fiber laser capable of generating NLPs. The laser utilized a ring resonator consisting of a 6-m length of $\mathrm{Yb}$-doped fiber, a nonlinear polarization evolution (NPE) port, an optical isolator and a long segment of single mode fibers (SMF28). An optical combiner was used to couple the signal and pumping light together before being sent into the active fiber. The doublecladding gain fiber had a $10-\mu \mathrm{m}$ core (LIEKKI, Yb1200-10/125DC). Pumping was performed by multi-mode laser diodes $(\lambda=915 \mathrm{~nm})$. The NPEport consisted of a half-wave plate (HWP), quarterwave plates (QWP) and a polarization beam splitter (PBS).

The ANDi Yb-doped fiber laser, i.e., the seed laser, generated NLPs with an average power of $200 \mathrm{~mW}$ (pulse energy of $62.5 \mathrm{~nJ}$ ). The NLPs 


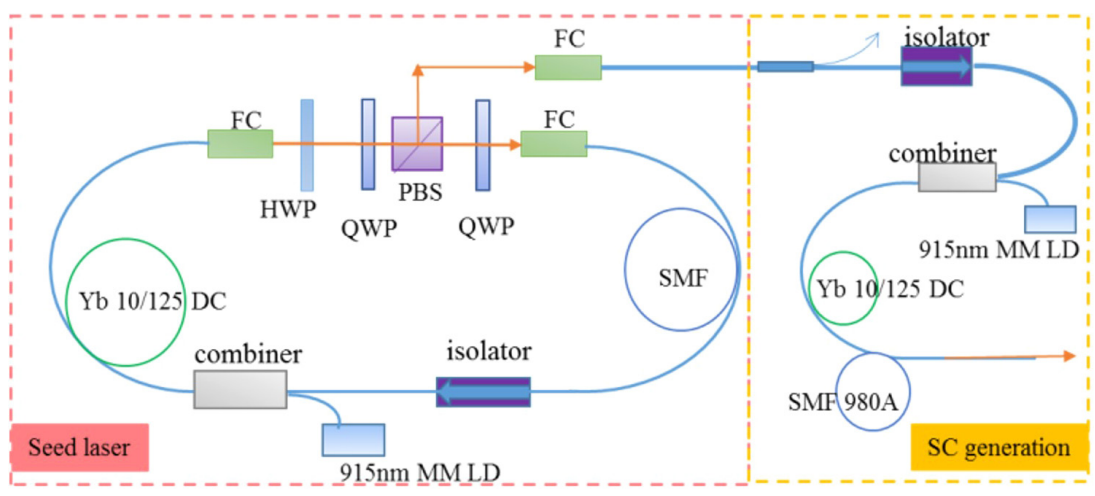

Fig. 1. Schematic of the fiber laser system for generating SC. FC: fiber coupler; HWP: half-wave plate; QWP: quarter-wave plate; ISO: optical isolator; MM LD: multi-mode laser diodes.

exhibited a periodic pulse train at $3.2 \mathrm{MHz}$ with a spectral bandwidth of $83 \mathrm{~nm}$ and a double-scaled intensity autocorrelation trace with FWHM of $100 \mathrm{ps}$ and $250 \mathrm{fs}$ for the pedestal and spike, respectively. Output of the seed laser was coupled into a 2-m length of $\mathrm{Yb}$-doped fiber (LIEKKI, Yb120010/125DC) also pumped with a 915-nm laser diode. The amplified NLPs were then used to excite the SC in a 50-m length of standard single mode fiber (POFC, SMF980A). At a pump power of $4.5 \mathrm{~W}$, we were able to generate a flat $\mathrm{SC}$ spectrum at the central wavelength $1320 \mathrm{~nm}$ with a spectral bandwidth up to $420 \mathrm{~nm}$ (in 3-dB bandwidth), as shown in Fig. 2. The average output power reached up to $560 \mathrm{~mW}$ (pulse energy of $175 \mathrm{~nJ}$ ). The spectral components of SC expanded to longer wavelength at higher pump power, mainly due to cascaded Raman scattering. ${ }^{19}$

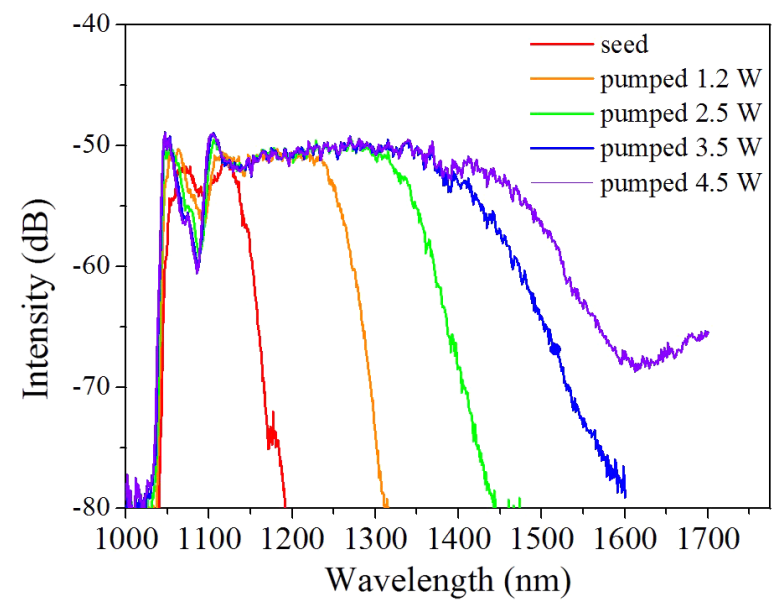

Fig. 2. The SC spectra generated by the amplified ANDi Yb-doped fiber laser.

\section{2. $\quad$ Imaging processing}

To examine the possible improvement of imaging quality by using the NLP-pumped SC source, some coefficients were used for quantitative comparison. In the previous studies, several blind speckle denoising metrics were used to quantify the imaging quality. They are SNR, CNR, ENL, and XCOR value.

These coefficients can be defined as follows ${ }^{21}$ :

$$
\begin{aligned}
& \mathrm{SNR}=10 \times \log _{10}\left(\frac{\operatorname{mean}\left[I_{(i, j)}^{2}\right]}{\sigma_{o m}^{2}}\right), \\
& \mathrm{CNR}=10 \times \log _{10}\left(\frac{\mu_{o m}-\mu_{b}}{\sqrt{\sigma_{o m}^{2}+\sigma_{b}^{2}}}\right), \\
& \mathrm{ENL}_{m}=\frac{\mu_{o m}^{2}}{\sigma_{o m}^{2}}, \\
& \mathrm{XCOR}=\frac{\sum_{(i, j)} I_{\text {before }}(i, j) I_{\mathrm{after}}(i, j)}{\sqrt{\sum_{(i, j)}\left[I_{\text {before }}(i, j)\right]^{2} \sum_{(i, j)}\left[I_{\text {after }}(i, j)\right]^{2}}},
\end{aligned}
$$

where $I_{(i, j)}$ is the intensity at location $(i, j)$ in the selected region of interest (ROI). $\sigma_{b}$ and $\mu_{b}$ are the standard deviations of the intensity and the mean of the intensity in the background region, respectively; $\mu_{o m}$ is the mean of the pixel values in the $m$ th ROI and $\sigma_{o m}$ is the pixel standard deviation. $I_{\text {before }}$ and $I_{\text {after }}$ are the intensity data before and after denoising, respectively; $(i, j)$ are the indices of the images; $\mu_{\text {after }}, \sigma_{\text {after }}, \mu_{\text {before }}$, and $\sigma_{\text {before }}$ are the mean and the standard deviation of the intensity in the processed OCT images and original OCT images, 


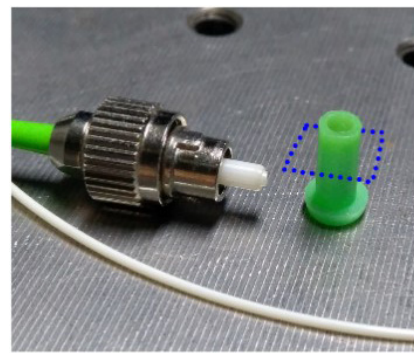

(a)

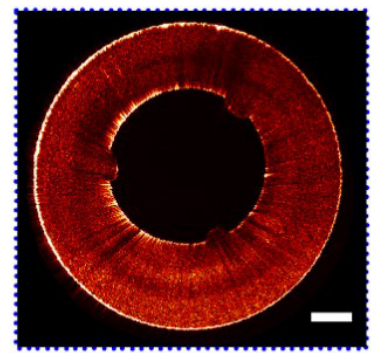

(b)
Fig. 3. (a) The picture of fiber connector. The blue dashed box illustrates the circular imaging plane. (b) SS-OCT crosssectional image of head cover of fiber connector. Scale bar represents $500 \mu \mathrm{m}$.

respectively. We will discuss in detail the physical meaning of SNR, CNR, ENL, and XCOR later.

In this experiment, we used a simple sample to demonstrate the imaging quality by applying SC generated by NLPs. As we know, it is relatively easier to see speckle than real signal in the uniform material and the coefficients can be calculated precisely. A sample with more structure would be more intuitive, but how its CNR is calculated is unclear. In order to illustrate our points quantitatively, we used a head cover of fiber connector as the sample, as shown in Fig. 3(a). The corresponding circularscan two-dimensional (2D) OCT image obtained with a conventional 1310-nm SS-OCT setup is shown in Fig. 3(b). In this image, there are two clear round-shaped boundaries, which indicate the plastic edge of the head cover. Between the two roundshaped boundaries, there are many bright spots. These bright spots apparently result from the speckle noise. Otherwise, because the material of the sample is uniform, there should exist no bright spot and the intensity distribution between two boundaries should be uniform. Because of the sample's uniformity, it does not matter which area is imaged, even though the three images are quite different. All three systems have different lateral resolution and axial resolution. Besides, each time we switch from one imaging system to another, we cannot ensure to image exactly the same place. Therefore, we finally choose the most stable region for comparison.

To compare the difference between random noise and speckle in OCT cross-sectional images, a simple averaging method to superimpose the consecutive B-scan images acquired at the same position can be used. The random noise can be reduced in the superimposed image while the speckle noise, as well as the structural signal, will remain with conventional OCT configurations. This is true for typical light sources such as SLED, swept laser sources or femtosecond lasers, because the speckle noise will remain even after image processing of superposition. In contrast, the amplitude, duration and even wavefront of NLP-based light sources are randomly varying from pulse to pulse. It is conceivable that the use of the NLP-pumped SC source may actually induce decorrelation of speckle noise from the sample and thus enable the speckle suppression by the simple method of averaging. Therefore, we utilized it as the light source in a conventional OCT configuration to get OCT images and then measured the denoising metrics of the original and the superimposed images. With the image processing of superposition, the random noise can be reduced and the image quality can be enhanced.

\section{Results and Discussion}

The SD-OCT image obtained by using the NLPpumped SC light source is shown in Fig. 4. The structure of fiber connector head cover is clearly visible. We may therefore further analyze the images in terms of the denoising metrics. In this study,

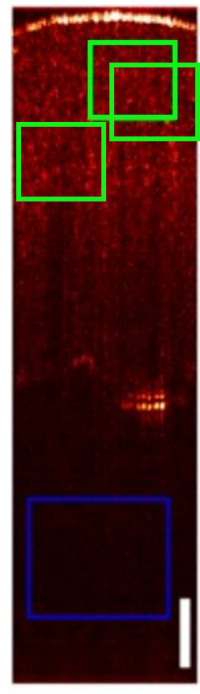

(a)

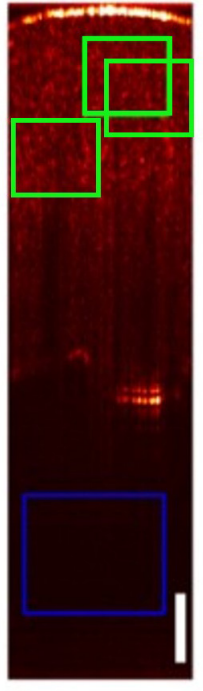

(b)

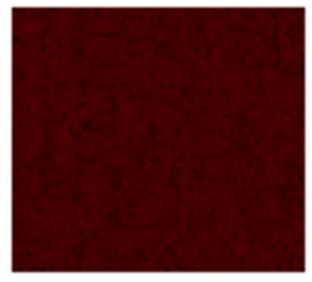

(c)

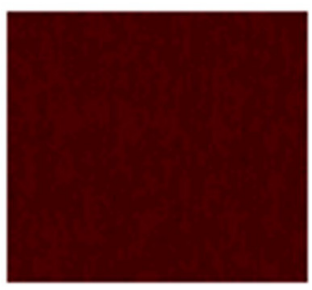

(d)
Fig. 4. (a) Single frame of head cover using SC pumped by NLPs. (b) Denoised image combined six successive images. The green boxes in these images mark three pairs of homogeneous ROIs used for calculation of SNR, CNR, and ENL, while the blue boxes mark regions of background that were used to obtain SNR and CNR. (c, d) Enlarged and log scaled image in one pair of green box in (a) and (b). Scale bar represents $200 \mu \mathrm{m}$. 
we actually use six frames and one is shown in Fig. 4(a). Each frame is measured in the same position and under the same conditions. Thereafter, the superposition of the six images is implemented, as shown in Fig. 4(b). Both Figs. 4(a) and 4(b) are shown in linear scale. We calculated SNR, CNR, and ENL by three different ROIs. All ROIs were circled by green boxes in Figs. 4(a) and 4(b). In order to observe the denoising effect more intuitively, we enlarge one pair of green box in Figs. 4(a) and 4(b), then present them in log scale in Figs. 4(c) and 4(d), respectively. In comparison with original images, the superimposed images not only enhance the signal intensity but also reduce the noise level. Furthermore, the intensity distribution between two arc-shaped boundaries is smoother than the previous results as shown in Fig. 3(b), indicating that using the SC generated by NLPs source in OCT systems is helpful to reduce the speckle noise and achieve high quality image.

To evaluate the performance of the light source, we compared three OCT light sources: (1) SC pumped by NLPs using a conventional SD-OCT system, with central wavelength of $1320 \mathrm{~nm}$, bandwidth of $420 \mathrm{~nm}$, lateral resolution of $10 \mathrm{um}$, and system SNR of $89 \mathrm{~dB}$. (2) A commercial SC source (Wuhan Yangtze Soton Laser Co., Ltd.) using the same SD-OCT system, with central wavelength of $1320 \mathrm{~nm}$, bandwidth of $300 \mathrm{~nm}$, lateral resolution of $10 \mathrm{um}$, and system SNR of $92 \mathrm{~dB}$. (3) Commercial swept source (HSL-2000, Santec Corp.) using a conventional SS-OCT system, with central wavelength of $1326 \mathrm{~nm}$, bandwidth of $108 \mathrm{~nm}$, lateral resolution of $10 \mathrm{um}$, and SNR of $110 \mathrm{~dB}$. Original OCT images and corresponding superimposed images are shown in Figs. 4-6, respectively. It is worth to note that all of the above-mentioned light sources are centered around $1310 \mathrm{~nm}$ and the OCT setup is independently utilized to get OCT images of the same sample under the same conditions.

The imaging quality metrics (SNR, CNR, ENL, XCOR) of single frame and the average of six frames obtained by using the three light sources are calculated and compared with each other, as summarized in Table 1. We choose three different pairs of ROIs to calculate SNR, CNR, and ENL. Their mean values and standard deviations are shown in Table 1 . The small standard deviations imply that the choice of ROIs does not affect the results very much. First, SNR measures the ratio between the average amplitude of the intensity in the ROI and

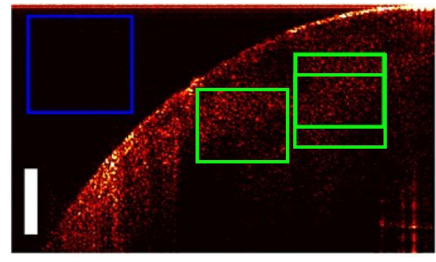

(a)

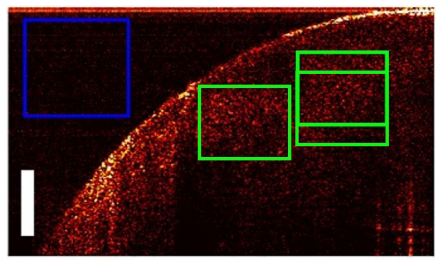

(b)

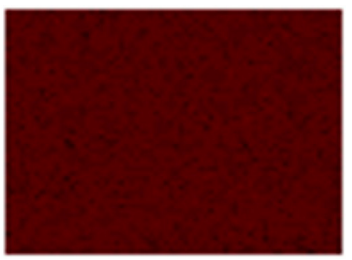

(c)

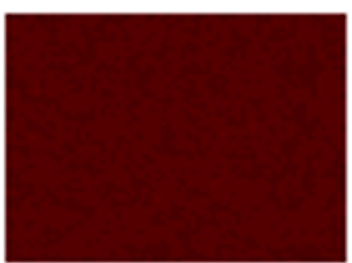

(d)
Fig. 5. (a) Single image of head cover using commercial SC. (b) Denoised image combined six successive images. The green boxes mark three pairs of the ROIs, while the blue boxes mark regions of background. (c, d) Enlarged and log scaled image in one pair of green box in (a) and (b). Scale bar represents $200 \mu \mathrm{m}$.

the standard deviation in the same region. As shown in Table 1, the improvement of SNR after image superposition is achieved for all the three light sources due to the noise suppression and speckle reduction. Second, CNR determines the contrast between the ROI and the area of background noise.

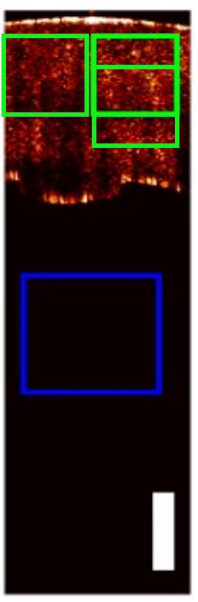

(a)

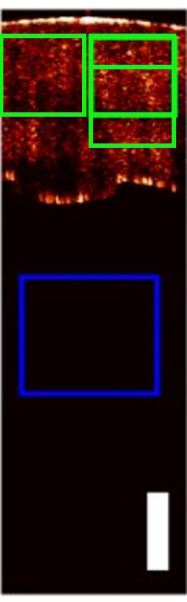

(b)

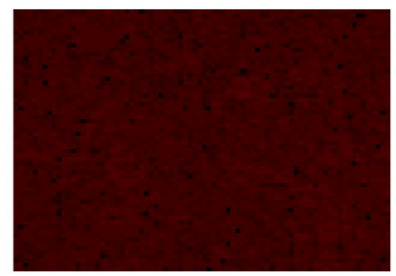

(c)

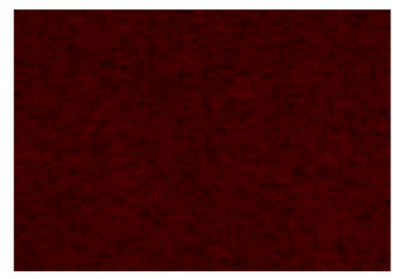

(d)
Fig. 6. (a) Single image of head cover using commercial SS. (b) Denoised image combined six successive images. The green boxes mark three pairs of the ROIs, while the blue boxes mark regions of background. (c, d) Enlarged and log scaled image in one pair of green box in (a) and (b). Scale bar represents $200 \mu \mathrm{m}$. 
Table 1. Quality metrics of speckle suppression for different OCT light sources.

\begin{tabular}{|c|c|c|c|c|c|c|}
\hline & \multicolumn{2}{|c|}{ SC pumped by NLPs } & \multicolumn{2}{|c|}{ Commercial SC } & \multicolumn{2}{|c|}{ Commercial SS } \\
\hline & Single frame & Denoised & Single frame & Denoised & Single frame & Denoised \\
\hline SNR (dB) & $6.4( \pm 0.1)$ & $9.7( \pm 0.3)$ & $6.6( \pm 0.1)$ & $9.6( \pm 0.4)$ & $5.6( \pm 0.1)$ & $6.0( \pm 0.1)$ \\
\hline CNR (dB) & $1.4( \pm 0.1)$ & $3.4( \pm 0.2)$ & $-1.1( \pm 0.2)$ & $0.7( \pm 0.3)$ & $2.0( \pm 0.1)$ & $2.3( \pm 0.1)$ \\
\hline ENL & $3.4( \pm 0.1)$ & $8.6( \pm 0.6)$ & $3.6( \pm 0.1)$ & $8.2( \pm 0.8)$ & $2.6( \pm 0.1)$ & $3.0( \pm 0.1)$ \\
\hline XCOR & & 0.794 & & 0.944 & & 0.985 \\
\hline
\end{tabular}

From Table 1, the CNR enhancement of our SC is $3.4-1.4=2 \mathrm{~dB}$, while that of the commercial SC and commercial swept source is $1.8 \mathrm{~dB}$ and $0.3 \mathrm{~dB}$, respectively. Thus for these metrics, the NLPpumped SC outperforms the other two light sources. Third, ENL measures the smoothness of the ROI which is homogeneous. Therefore, if the image is more homogenous, the coefficient of ENL is larger. The ENL becomes smaller when the homogenous appearance is corrupted by speckle pattern. As we can see, the ENL improvement of the NLP-pumped $C$ is $8.6-3.4=5.2$, while that of the commercial SC and commercial swept source is 4.6 and 0.4 , respectively. This indicates that the SC pumped by NLPs enables largest speckle suppression and achieves the best smoothness of ROI in superposition OCT image. Finally, XCOR is an indicator of the similarity between the denoised images and original images. In general, XCOR is usually smaller than 1 . But, it is very close to 1 when the denoised image is very similar to the original image. The commercial swept source has its XCOR of 0.985 , very close to 1 . This means that OCT images with the swept source have very low speckle decorrelation from frame to frame and the denoised image makes little change or improvement compared to the original image. Our custom-built SC has its XCOR of 0.794, indicating fairly good performance. As for the commercial SC, it has its XCOR of 0.944 very close to 1 , too. Compared to the original image with the commercial SC, we find that its denoised image after averaging does show some changes and improvement. This is mainly attributed to the reduction of the large intensity fluctuation of the commercial SC after denoising instead of speckle suppression. The large intensity fluctuation increases the mean intensity of the background and hence reduces the difference of mean intensity value between ROI and background region. In the same time, it increases the standard deviation of ROI and background. According to Eq. (2), the numerator is decreased and the denominator is increased, which may lead to $\left(\mu_{o m}-\mu_{b}\right) / \sqrt{\sigma_{o m}^{2}+\sigma_{b}^{2}}$ less than 1. So in Table 1, we get a negative value of CNR. Furthermore, the improvement of CNR, ENL, and XCOR after denoising with the commercial SC and commercial SS is not as significant as that with our SC. In summary, all light sources show the improvement of SNR using multi-frame averaging. Significantly, SC pumped by NLPs performs better as far as speckle reduction is concerned than other light sources, judging from the CNR, ENL, and XCOR. With the same SD-OCT setup, almost all the imaging quality metrics of our SC are better than the commercial SC source. Especially for the image averaged over six frames, the ENL of our SC is the largest one and the XCOR is the smallest, indicating best smoothness of ROI in OCT image. Therefore, SC pumped by NLPs has very good performance in terms of suppressing speckle noise while preserving image details. This is very important for biomedical imaging.

\section{Conclusion}

To conclude, we have demonstrated that the unique properties of SC pumped by NLPs can be combined with simple multi-frame compounding method for OCT speckle reduction. Compared with conventional light sources, our approach is more favorable for the decorrelation of successive B-scans and the speckle suppression in terms of image quality metrics of SNR, CNR, ENL, and XCOR value. Because our custom-built SC light source is based on fiber technology, the system is stable, compact, relatively inexpensive, and easy to connect with OCT system. We believe that it will be very promising to be used as a new light source for ultrahigh resolution OCT imaging and other applications as well. 


\section{Conflict of Interest}

The authors have no relevant financial interests in this article and no potential confilicts of interest to disclose.

\section{Acknowledgments}

This research was supported by the National Natural Science Foundation of China (61227807, 61575108 and 61505034), Tsinghua Initiative Scientific Research Program (2013THZ02-3). C. L. Pan and Y. J. You were supported in part by the Ministry of Science and Technology, Taiwan, under Grant 103-2622-E-007-006-CC2 and by the National Tsing Hua University Research Program Grant 104N2711E1. C.L. Pan is also supported by the Air Force Office of Scientific Research FA238613-1-4086. The first three authors contribute equally to this paper.

\section{References}

1. J. M. Schmitt, S. H. Xiang, K. M. Yung, "Speckle in optical coherence tomography," J. Biomed. Opt. 4, 95-105 (1999).

2. M. Bashkansky, J. Reintjes, "Statistics and reduction of speckle in optical coherence tomography," Opt. Lett. 25, 545-547 (2000).

3. J. M. Schmitt, "Array detection for speckle reduction in optical coherence microscopy," Phys. Med. Biol. 42, 1427 (1997).

4. C. Dongyao, B. En, L. Yuemei et al., "Multifiber angular compounding optical coherence tomography for speckle reduction," Opt. Lett. 42(1), 125-128 (2017).

5. B. F. Kennedy, T. R. Hillman, A. Curatolo, D. D. Sampson, "Speckle reduction in optical coherence tomography by strain compounding," Opt. Lett. 35, 2445-2447 (2010).

6. M. Pircher, E. Go, R. Leitgeb, A. F. Fercher, C. K. Hitzenberger, "Speckle reduction in optical coherence tomography by frequency compounding," J. Biomed. Opt. 8, 565-569 (2003).

7. C. Magnain, H. Wang et al., "En face speckle reduction in optical coherence microscopy by frequency compounding," Opt. Lett. 41(9), 1925 (2016).

8. A. Ozcan, A. Bilenca, A. E. Desjardins, B. E. Bouma, G. J. Tearney, "Speckle reduction in optical coherence tomography images using digital filtering," J. Opt. Soc. Am. A. 24, 1901-1910 (2007).

9. D. C. Adler, T. H. Ko, J. G. Fujimoto, "Speckle reduction in optical coherence tomography images by use of a spatially adaptive wavelet filter," Opt. Lett. 29, 2878-2880 (2004).

10. A. Wax, E. T. Jelly, K. K. Chu et al., "Real-time speckle reduction in optical coherence tomography using the dual window method," Biomed. Opt. Express 9(2), 616 (2018).

11. P. Puvanathasan, K. Bizheva, "Speckle noise reduction algorithm for optical coherence tomography based on interval type II fuzzy set," Opt. Express 15, 15747-15758 (2007).

12. Y. Hancheng, G. Jianlin, L. Aiting, "PNLM: A probability-based non-local means filter for speckle noise suppression in optical coherence tomography images," Opt. Lett. 41(5), 994 (2016).

13. C. Cuartas-Vélez, R. Restrepo, B. E. Bouma et al., "Volumetric non-local-means based speckle reduction for optical coherence tomography," Biomed. Opt. Express 9(7), 3354-3374 (2018).

14. K. Yu, L. Ji, L. Wang, P. Xue, "How to optimize OCT image," Opt. Express 9, 24-35 (2001).

15. A. Wong, A. Mishra, K. Bizheva, D. A. Clausi, "General Bayesian estimation for speckle noise reduction in optical coherence tomography retinal imagery," Opt. Express 18, 8338-8352 (2010).

16. O. Liba, M. D. Lew, E. D. SoRelle, R. Dutta, D. Sen, D. M. Moshfeghi, S. Chu, A. de la Zerda, "Specklemodulating optical coherence tomography in living mice and humans," Nat. Commun. 8, 15845 (2017).

17. D. Y. Tang, L. M. Zhao, B. Zhao, "Soliton collapse and bunched noise-like pulse generation in a passively mode-locked fiber ring laser," Opt. Express 13, 2289-2294 (2005).

18. J. C. Hernandez-Garcia, O. Pottiez, J. M. EstudilloAyala, "Supercontinuum generation in a standard fiber pumped by noise-like pulses from a figure-eight fiber laser," Laser Phys. 22, 221-226 (2012).

19. A. Zaytsev, C. Lin, Y. You, C. Chung, C. Wang, C. Pan, "Supercontinuum generation by noise-like pulses transmitted through normally dispersive standard single-mode fibers," Opt. Express 21, 16056-16062 (2013).

20. Y. You, C. Wang, Y. Lin, A. Zaytsev, P. Xue, C. Pan, "Ultrahigh-resolution optical coherence tomography at $1.3 \mu \mathrm{m}$ central wavelength by using a supercontinuum source pumped by noise-like pulses," Laser Phys. Lett. 13, 25101 (2016).

21. D. Yin, Y. Gu, P. Xue, "Speckle-constrained variational methods for image restoration in optical coherence tomography," JOSA A. 30, 878-885 (2013). 